\title{
Temporal changes of COVID-19 pneumonia by mass evaluation using CT: a retrospective multi-center study
}

\author{
Chao Wang ${ }^{1}$, Peiyu Huang ${ }^{1}$, Lihua Wang ${ }^{1}$, Zhujing Shen ${ }^{1}$, Bin Lin ${ }^{1}$, Qiyuan Wang ${ }^{1}$, Tongtong Zhao ${ }^{2}$, \\ Hanpeng Zheng ${ }^{3}$, Wenbin Ji ${ }^{4}$, Yuantong Gao ${ }^{5}$, Junli $\mathrm{Xia}^{6}$, Jianmin Cheng ${ }^{7}$, Jianbing $\mathrm{Ma}^{8}$, Jun Liu ${ }^{9}$, \\ Yongqiang Liu ${ }^{10}$, Miaoguang Su${ }^{11}$, Guixiang Ruan ${ }^{12}$, Jiner Shu ${ }^{13}$, Dawei Ren ${ }^{14}$, Zhenhua Zhao ${ }^{15}$, \\ Weigen Yao ${ }^{16}$, Yunjun Yang ${ }^{17}$, Bo Liu ${ }^{18,19}$, Minming Zhang ${ }^{1}$
}

${ }^{1}$ Department of Radiology, The Second Affiliated Hospital, Zhejiang University School of Medicine, Hangzhou, China; ${ }^{2}$ Department of Radiology, The Second People's Hospital of Fuyang, Fuyang, China; ${ }^{3}$ Department of Radiology, Yueqing People's Hospital, Yueqing, Wenzhou, China; ${ }^{4}$ Department of Radiology, Taizhou Hospital of Zhejiang Province, Taizhou, China; ${ }^{5}$ Department of Radiology, Ruian People's Hospital, The Third Affiliated Hospital of Wenzhou Medical University, Ruian, China; ${ }^{6}$ Bozhou Bone Trauma Hospital Image Center, Bozhou, China; ${ }^{7}$ Department of Radiology, The Second Affiliated Hospital and Yuying Children's Hospital of Wenzhou Medical University, Wenzhou, China; ${ }^{8}$ Department of Radiology, The First Hospital of Jiaxing, Affiliated Hospital of Jiaxing University, Jiaxing, China; ${ }^{9}$ Department of Radiology, The Second Xiangya Hospital, Central South University, Changsha, China; ${ }^{10}$ Department of Radiology, Kecheng People's Hospital, Quzhou, China; ${ }^{11}$ Department of Radiology, The People's Hospital of Pingyang, Pingyang Hospital Affiliated to Wenzhou Medical University, Pingyang, China; ${ }^{12}$ Department of Radiology, The First People's Hospital of Yuhang District, Hangzhou, China; ${ }^{13}$ Department of Radiology, Jinhua Hospital of Zhejiang University, Jinhua Municipal Central Hospital, Jinhua, China; ${ }^{14}$ Department of Radiology, Ningbo First Hospital, Ningbo, China; ${ }^{15}$ Department of Radiology, Shaoxing People's Hospital, Shaoxing, China; ${ }^{16}$ Department of Radiology, Yuyao People's Hospital, The Affiliated Yangming Hospital of Ningbo University, Ningbo, China; ${ }^{17}$ Department of Radiology, The First Affiliated Hospital of Wenzhou Medical University, Wenzhou, China; ${ }^{18}$ Shanghai Key Laboratory of Artificial Intelligence for Medical Image and Knowledge Graph, Shanghai, China; ${ }^{19}$ YITU AI Research Institute for Healthcare, Shanghai, China

Contributions: (I) Conception and design: C Wang, M Zhang; (II) Administrative support: M Zhang; (III) Provision of study materials or patients: T Zhao, H Zheng, W Ji, Y Gao, J Xia, J Cheng, J Ma, J Liu, Y Liu, M Su, G Ruan, J Shu, D Ren, Z Zhao, W Yao, Y Yang; (IV) Collection and assembly of data: L Wang, Z Shen, B Lin, Q Wang; (V) Data analysis and interpretation: C Wang, P Huang, B Liu; (VI) Manuscript writing: All authors; (VII) Final approval of manuscript: All authors.

Correspondence to: Minming Zhang. Department of Radiology, the Second Affiliated Hospital, Zhejiang University School of Medicine, No. 88 Jiefang Road, Hangzhou, China. Email: zhangminming@zju.edu.cn.

Background: Coronavirus disease 2019 (COVID-19) has widely spread worldwide and caused a pandemic. Chest CT has been found to play an important role in the diagnosis and management of COVID-19. However, quantitatively assessing temporal changes of COVID-19 pneumonia over time using CT has still not been fully elucidated. The purpose of this study was to perform a longitudinal study to quantitatively assess temporal changes of COVID-19 pneumonia.

Methods: This retrospective and multi-center study included patients with laboratory-confirmed COVID-19 infection from 16 hospitals between January 19 and March 27, 2020. Mass was used as an approach to quantitatively measure dynamic changes of pulmonary involvement in patients with COVID-19. Artificial intelligence (AI) was employed as image segmentation and analysis tool for calculating the mass of pulmonary involvement.

Results: A total of 581 confirmed patients with 1,309 chest CT examinations were included in this study. The median age was 46 years (IQR, 35-55; range, 4-87 years), and 311 (53.5\%) patients were male. The mass of pulmonary involvement peaked on day 10 after the onset of initial symptoms. Furthermore, the mass of pulmonary involvement of older patients $(>45$ years) was significantly severer $(\mathrm{P}<0.001)$ and peaked later (day 11 vs. day 8 ) than that of younger patients ( $\leq 45$ years). In addition, there were no significant differences in the peak time (day 10 vs. day 10) and median mass $(\mathrm{P}=0.679)$ of pulmonary involvement between male and female. 
Conclusions: Pulmonary involvement peaked on day 10 after the onset of initial symptoms in patients with COVID-19. Further, pulmonary involvement of older patients was severer and peaked later than that of younger patients. These findings suggest that AI-based quantitative mass evaluation of COVID-19 pneumonia hold great potential for monitoring the disease progression.

Keywords: Coronavirus disease 2019 (COVID-19); chest CT; temporal changes; artificial intelligence (AI)

Submitted May 16, 2020. Accepted for publication Jun 28, 2020.

doi: $10.21037 / \mathrm{atm}-20-4004$

View this article at: http://dx.doi.org/10.21037/atm-20-4004

\section{Introduction}

Coronavirus Disease 2019 (COVID-19) has officially been declared a pandemic by the World Health Organization (WHO), which was ultimately found to be caused by a new severe acute respiratory syndrome coronavirus 2 (SARSCov-2) (1). This virus has spread to over 200 countries, with more than 5,000,000 cases and over 300,000 deaths reported worldwide as of 24 May, 2020.

Chest CT plays a central role in monitoring the disease severity of COVID-19 pneumonia to guide clinical management (2). Ground-glass opacities (GGO) and consolidations are two most common CT features of COVID-19 pneumonia, which are affected by the amount of exudation in the pathological process and may transform into each other over time (3). COVID-19 pneumonia presents as chest lesions with rapid evolution from focal unilateral to diffuse bilateral GGO that progressed to or co-existed with consolidations within several weeks (4). Because of the co-existed different changes in the number/size and density of lung lesions (5), quantitatively measuring temporal changes of COVID-19 pneumonia can be challenging. Several studies investigated the temporal changes of COVID-19 pneumonia, but these studies assessed imaging findings using CT images all by radiologists' subjective experience (2,5-9), which were unable to accurately and quantitatively evaluate the disease severity with large inter- and intra-observer variability. To date, quantitatively assessing dynamic changes of COVID-19 pneumonia over time has still not been fully elucidated.

Diameter measurement and volumetric measurement are two common parameters for evaluating the changes in lung lesions $(10,11)$. However, neither diameter measurement nor volumetric measurement can accurately and quantitatively evaluate the progression from GGO lesions to consolidation lesions. Mass is a parameter that integrates volume and density: mass increases if the volume of a lesion increases or if its density increases. Mass can be calculated by multiplying nodule volume and density because $\mathrm{X}$-ray attenuation values are proportional to tissue density (i.e., mass per unit volume) (12). In fact, mass measurement has been confirmed to enable detection of growth of pulmonary ground-glass nodules earlier and is subject to less variability than are diameter or volumetric measurement (13). Therefore, in the present study, we introduced mass estimation as a method for quantitatively evaluating temporal changes of COVID-19 pneumonia. In addition, it is now recognized that artificial intelligence (AI) holds great promise for segmentating and quantifying pneumonia lesions $(14,15)$. In this study, to investigate temporal changes of COVID-19 pneumonia, we further introduced $\mathrm{AI}$ to quantitatively measure mass of pulmonary involvement in patients with COVID-19. We present the following article in accordance with the STROBE reporting checklist (available at http://dx.doi.org/10.21037/atm-204004).

\section{Methods}

\section{Patients}

The study was conducted in accordance with the Declaration of Helsinki (as revised in 2013). This retrospective multicenter study was approved by the Institutional Review Board of each participating hospital. The written informed consent was waived. This study included 1491 chest CT examinations from 649 patients with laboratory-confirmed COVID-19 at 16 hospitals between January 19 and March 27, 2020. The exclusion criteria included: (I) unknown date of initial symptoms onset; (II) without initial symptoms; (III) scans with slice thickness $>5 \mathrm{~mm}$; (IV) multiple calcification. After applying the exclusion criteria, 1,309 chest CT examinations from 581 patients were selected in this study 


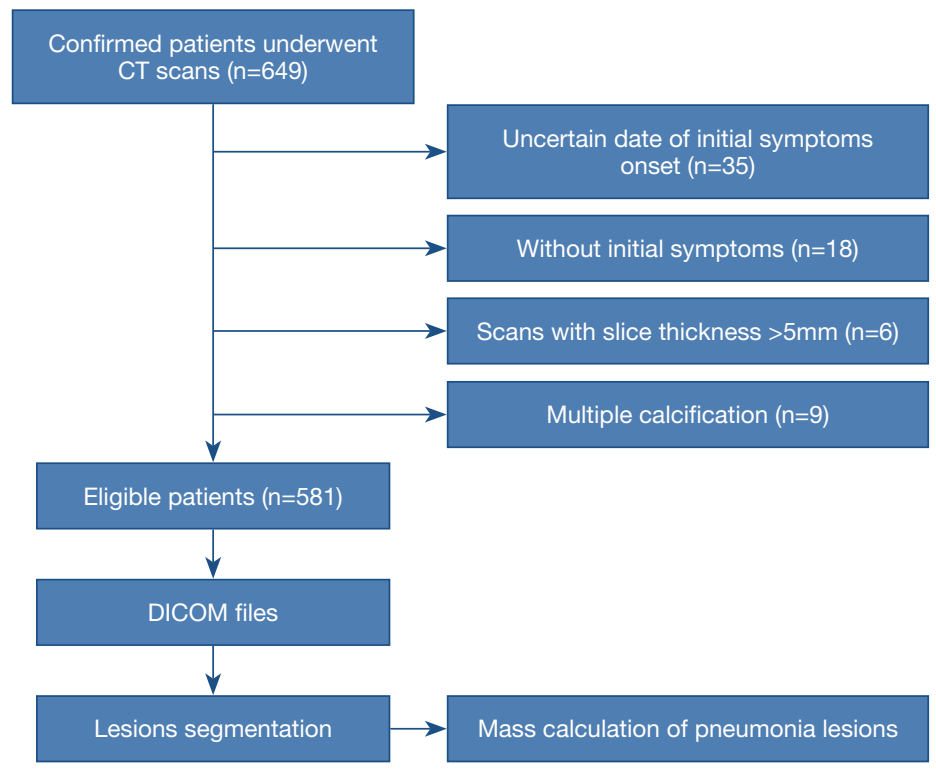

Figure 1 Flow diagram of the study population.

(Figure 1). Treatment criteria for patients with COVID-19 pneumonia conformed to the Guidelines for the Diagnosis and Treatment of COVID-19 Pneumonia published by the National Health Commission of the People's Republic of China (http://www.nhc.gov.cn/yzygj/s7653p/202002/8334 a8326dd94d329df351d7da8aefc2.shtml). The median CT follow-up period was 10 days from symptoms onset (IQR, 6-16; range, 0-58 days). The number distribution of CT follow-up examinations was summarized in Figure S1. All patients were recovered after treatment and none of patients were died.

\section{CT protocol}

CT examinations were performed using 16-slice to 128-slice CT scanners of different manufacturers, including SIEMENS, GE, TOSHIBA, and PHILIPS. Chest CT examinations were performed with a varying slice-thickness from 1 to $5 \mathrm{~mm}$. Standard lung algorithm settings were used as follows: tube voltage, $100-130 \mathrm{kV}$; tube current, 100-440 mA; section thickness, 1 to $5 \mathrm{~mm}$; reconstruction matrix, $512 \times 512$.

\section{AI-based quantization and mass calculation}

The Quantitative Evaluation System of CT for COVID-19 (YT-CT-Lung, YITU Healthcare Technology Co., Ltd., China) (Figure S2) was employed as the image automatic segmentation and analysis tool under supervision of two experienced radiologists ( $>5$ years of experience in chest imaging diagnosis) (16). This system combined a fully convolutional network with adaptive thresholding and morphological operations for segmentation of lungs and pneumonia lesions (17). After segmentation, the total volume and mean CT value of pneumonia lesions were quantitatively computed by AI. CT value in Hounsfield units can be translated directly into physical density in milligrams per milliliter by adding 1,000 to the Hounsfield unit value (12). The mass within the volume of pneumonia lesions, as segmentated on all sections that contained pneumonia lesions, was calculated by multiplying total lesions volume by mean lesions density (i.e., mean CT value $+1,000)(12,13)$. Our goal is to quantitatively temporal changes in mass of pulmonary involvement in patients with COVID-19 pneumonia. Figure 2 showed temporal changes in mass of pulmonary involvement in a 29-year-old woman with COVID-19 pneumonia.

\section{Statistical analysis}

The day of initial symptom onset was defined as illness day 0 . Continuous variables were expressed as the medians with interquartile ranges (IQR). The median values of lesions mass in all COVID-19 patients were plotted as a scatter plot over time. The median age of the patients was 46 years in this study, thus we further divided the patients into two 

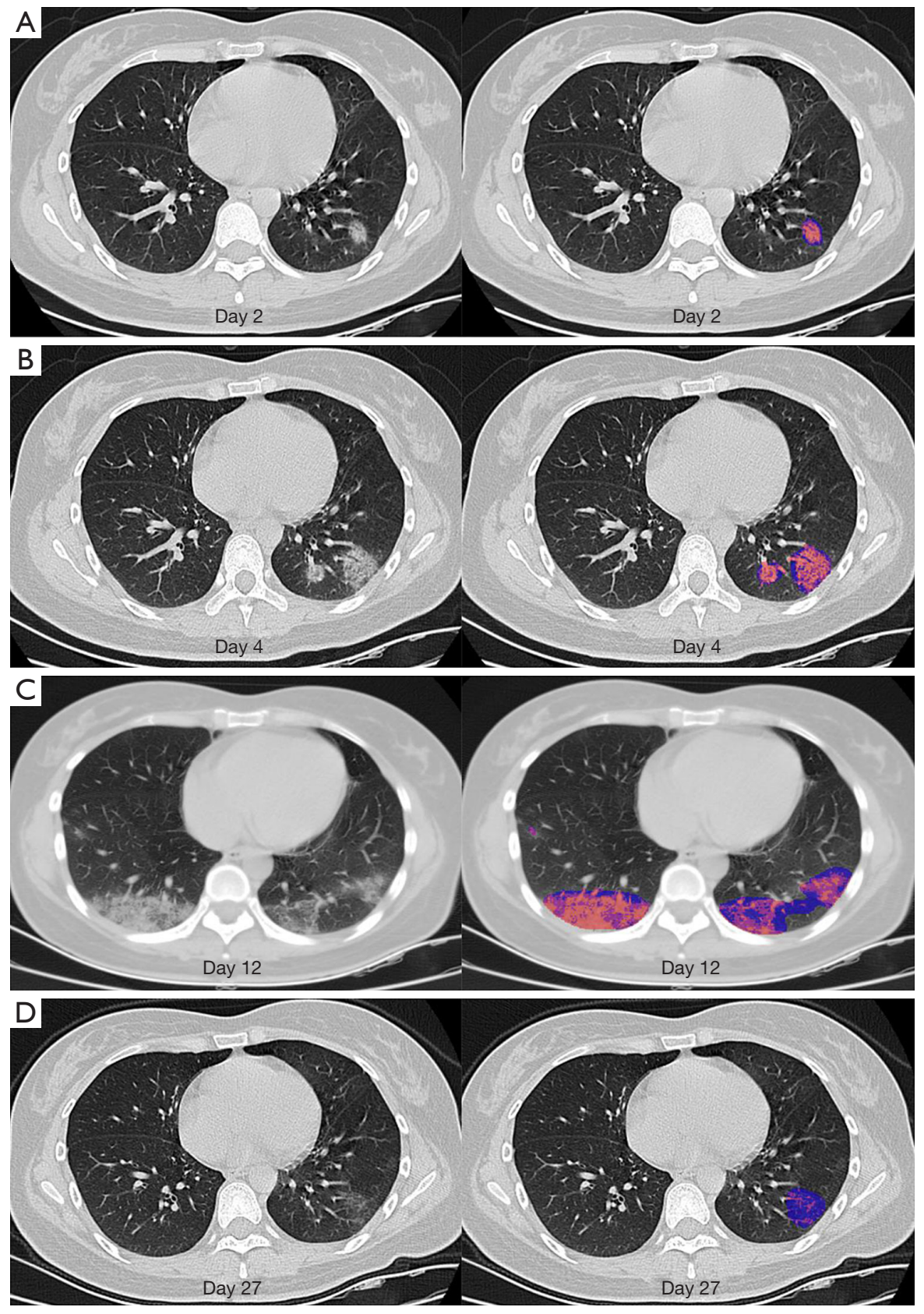

Figure 2 Dynamic changes in mass of pulmonary involvement in a 29-year-old woman with COVID-19 pneumonia presenting with persistent fever $\left(38.0^{\circ} \mathrm{C}\right.$ ) for two days. (A) At presentation (day 2), a small nodule of GGO with partial consolidation was demonstrated in the left lower lobe; after segmentation, the volume mean CT value of lesions were calculated by artificial intelligence (AI). Day 2, the volume of lesion was $6.51 \mathrm{~cm}^{3}$ and mean CT value of lesion was $-487.08 \mathrm{HU}$. Mass $=6.51 \times(-487.08+1000)=3339$. (B) Scan obtained on day 4 showed semi-consolidation with increased extent with superimposed inter- and intralobular septal thickening (crazy-paving pattern); day 4 , the volume of lesions was $12.05 \mathrm{~cm}^{3}$ and mean CT value of lesions was $-355.86 \mathrm{HU}$. Mass $=12.05 \times(-355.86+1000)=7762$. (C) Scan obtained on day 12 showed multiple semi-consolidation with increased extent in both lower lobe; day 12, the volume of lesions was $206.19 \mathrm{~cm}^{3}$ and mean CT value of lesions was -449.08 HU. Mass $=206.19 \times(-449.08+1000)=113594$. (D) Scan obtained on day 27 showed obvious absorption of abnormalities and only small pure GGO could be observed in the left lower lobe; day 27, the volume of lesion was $9.42 \mathrm{~cm}^{3}$ and mean CT value of lesion was -637.33 HU. Mass $=9.42 \times(-637.33+1000)=3416$. COVID-19, coronavirus disease 2019; GGO, ground-glass opacity. 
Table 1 The demographic characteristics, initial symptoms and clinical outcomes of the included patients

\begin{tabular}{lc}
\hline Variable & $\begin{array}{c}\text { Number of patients } \\
\text { (percentage) }\end{array}$ \\
\hline Median [interquartile range] age (years) & $46[35-55]$ \\
Gender & 311 \\
Male & 270 \\
Female & \\
Initial symptoms & $449(77.3 \%)$ \\
Fever & $275(47.3 \%)$ \\
Cough & $60(10.3 \%)$ \\
Sore throat & $52(8.9 \%)$ \\
Fatigue & $42(7.2 \%)$ \\
Chest distress & $34(5.9 \%)$ \\
Chills & $34(5.9 \%)$ \\
Sore muscle & $26(4.5 \%)$ \\
Headache or dizziness & $24(4.1 \%)$ \\
Diarrhea & $16(2.8 \%)$ \\
Running nose & $8(1.4 \%)$ \\
Anorexia & \\
Recovery & \\
\hline & \\
Died & $(0 \%)$ \\
\hline
\end{tabular}

groups ( $\geq 46$ and $\leq 45$ years). Then, the median values of lesions mass in the patients $\leq 45$ and $\geq 46$ years were also plotted as the scatter plot over time, respectively. The median mass values of pneumonia lesions were compared between groups using Kruskal-Wallis tests and MannWhitney U tests. Statistical analyses were performed using the $\mathrm{R}$ software package, version 3.6.2. $\mathrm{P}<0.05$ was considered to be statistically significant.

\section{Results}

A total of 581 confirmed patients with 1,309 chest CT examinations were included in this study (Figure 1). Demographic and clinical characteristics of 581 patients with COVID-19 were summarized in Table 1 . The median age was 46 years (IQR, 35-55 years; range, 4-87 years), and $311(53.5 \%)$ patients were male. The most common presenting symptoms were fever $(77.3 \%)$ and cough (47.3\%).

Figure $3 A$ showed temporal changes in mass of pneumonia lesions over weeks. There were significant differences between the groups of different weeks (KruskalWallis test, $\mathrm{P}<0.001)$. The mass of pneumonia lesions peaked on week 2 with the median values of 87952 (IQR, 24,222-185,326, Mann-Whitney U test, $\mathrm{P}<0.001$, compared to that of week 1). After that, the mass of pneumonia lesions significantly declined on week 3 with the median values of 61,194 (IQR, 15,742-147,507, $\mathrm{P}=0.007$, compared to that
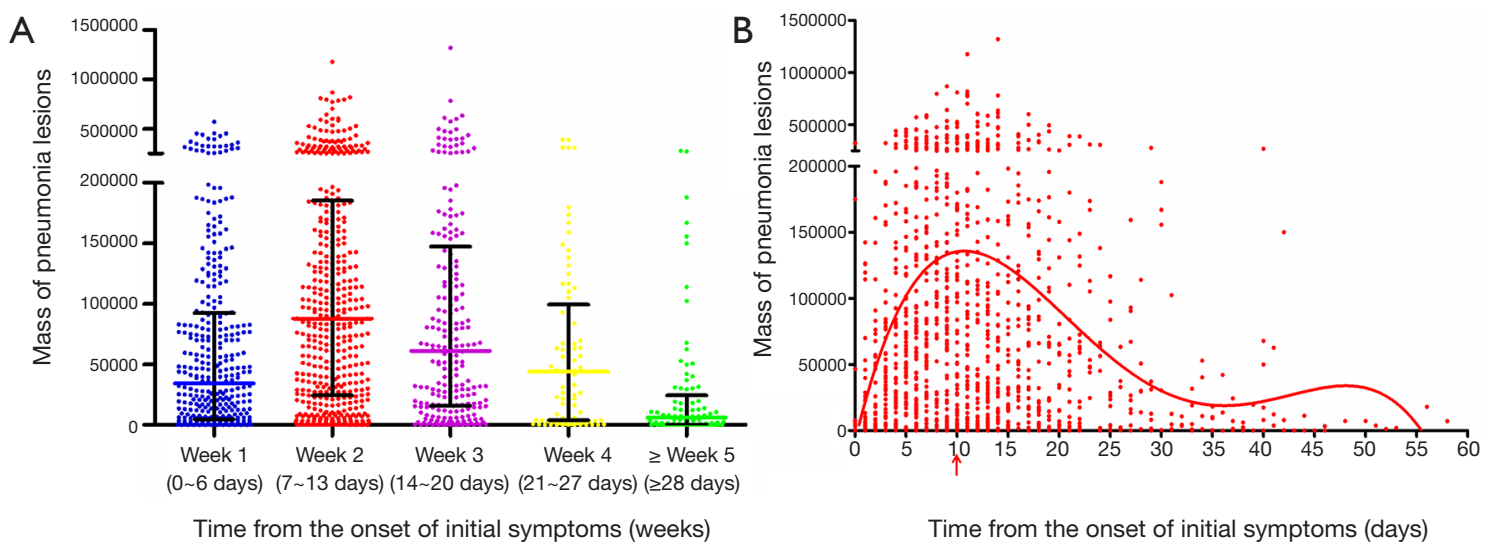

Figure 3 Dynamic changes in mass of pulmonary involvement over time after the onset of initial symptoms. (A) Dynamic changes in mass of pulmonary involvement over weeks. Peak in mass of pulmonary involvement occurred on week 2. Coloured bars represent medians and black bars represent interquartile ranges. (B) Dynamic changes in mass of pulmonary involvement over days. Peak in mass of pulmonary involvement occurred on day 10 (curve fitting equation: $y=-0.4301 \times x^{4}+54.45 \times x^{3}-2272 \times x^{2}+32018 x-7808$, in which $x=$ day from the onset of initial symptoms, $\mathrm{y}=$ total mass of the pneumonia lesions; $\mathrm{R}^{2}=0.08, \mathrm{P}<0.001$ ). 

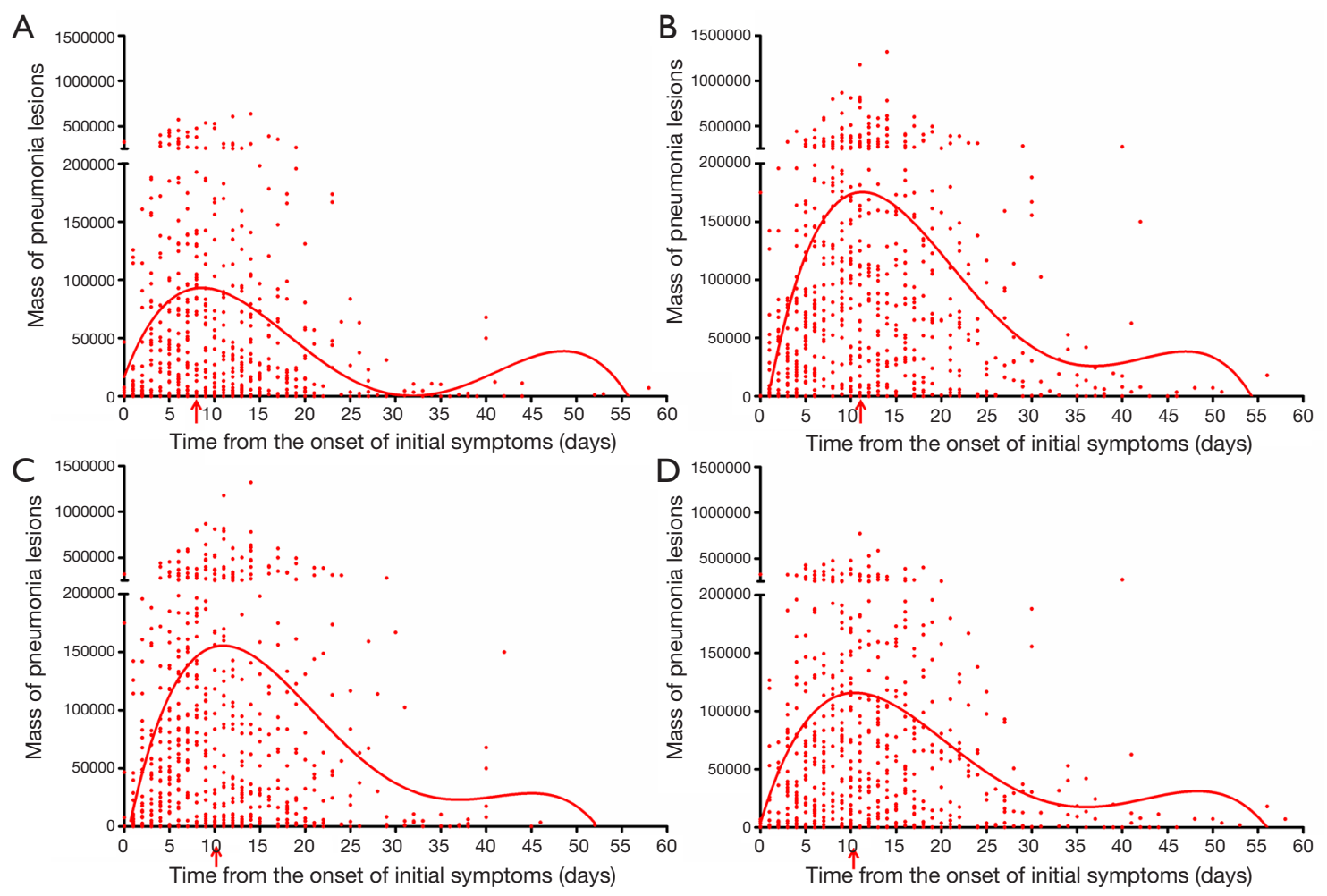

Figure $4(\mathrm{~A}, \mathrm{~B})$ Dynamic changes in mass of involved pneumonia lesions from the onset of initial symptoms in the group of patients $\leq 45$ and $\geq 46$ years, respectively. (A) In the group of patients $\leq 45$ years, the peak in mass of pulmonary involvement occurred on day 8 (red arrow) (curve fitting equation: $y=-0.3906 \times x^{4}+46.35 \times x^{3}-1748 \times x^{2}+20762 x+16527$, in which $x=$ day from the onset of initial symptoms, $y=$ mass of the pneumonia lesions; $\mathrm{R}^{2}=0.07, \mathrm{P}<0.001$ ); (B) in the group of patients $\geq 46$ years, the peak in mass of pulmonary involvement occurred on day 11 (red arrow) (curve fitting equation: $y=-0.5749 x x^{4}+73.15 \times x^{3}-3099 \times x^{2}+45349 x-37890$, in which $x=$ day from the onset of initial symptoms, $y=$ mass of the pneumonia lesions; $\left.\mathrm{R}^{2}=0.11, \mathrm{P}<0.001\right)$. (C,D) Dynamic changes in mass of involved pneumonia lesions from the onset of initial symptoms in the group of male and female patients, respectively. (C) In the group of male patients, the peak in mass of pulmonary involvement occurred on day 10 (red arrow) (curve fitting equation: $y=-0.5323 \times x^{4}+65.99 \times x^{3}-2729 \times x^{2}+38794 x-20950$, in which $x=$ day from the onset of initial symptoms, $y=$ mass of the pneumonia lesions; $\mathrm{R}^{2}=0.08, \mathrm{P}<0.001$ ); (D) in the group of female patients, the peak in mass of pulmonary involvement occurred on day 10 (red arrow) (curve fitting equation: $y=-0.3505 \times x^{4}+44.29 \times x^{3}-1837 \times x^{2}+25470 x+3803$, in which $x$ $=$ day from the onset of initial symptoms, $\mathrm{y}=$ mass of the pneumonia lesions; $\mathrm{R}^{2}=0.1, \mathrm{P}<0.001$ ).

of week 2), and further significantly declined on week 4 with the median values of 44,061 (IQR, 3,874-99,480, $\mathrm{P}=0.015$, compared to that of week 3 ). After week 4 ( $\geq 28$ days), pneumonia lesions of most patients were dissipated with the median values of 6,065 (IQR, 332-24,425, $\mathrm{P}<0.001$, compared to that of week 4 ). In addition, Figure $3 B$ showed temporal changes in mass of pneumonia lesions over days. The mass of pneumonia lesions peaked on day 10 after the onset of initial symptoms, and then gradually declined.

In this study, the median age of the patients was 46 years. Then, we further divided the patients into two groups ( $\geq 46$ years and $\leq 45$ years). In the group of patients $\leq 45$ years, the mass of pneumonia lesions peaked at 8 days after the onset of initial symptoms, and then gradually declined (Figure $4 A$ ). In the group of patients $\geq 46$ years, the mass of pneumonia lesions peaked at 11 days after the onset of initial symptoms, and then gradually declined (Figure 4B). In addition, temporal changes in mass of pneumonia lesions over days were plotted in women patients (Figure 4C) and men patients (Figure 4D), respectively. The peak in mass of pneumonia lesions both occurred on day 10 in male and female patients.

In addition, the mass of pneumonia lesions in the patients $\geq 46$ years (median values, 76,499; IQR, 20,583-168,766) were significantly greater than that of the patients $\leq 45$ years (median values, 26,526; IQR, 2,973-91,520) $(\mathrm{P}<0.001$, 

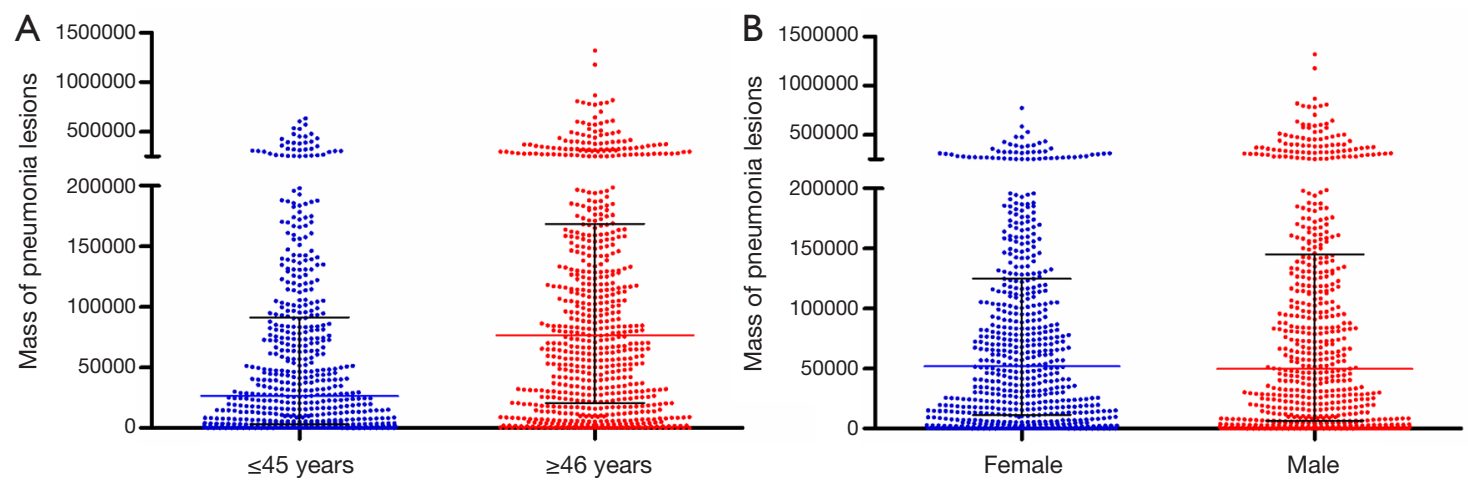

Figure 5 Association between age (A) and sex (B) and the mass of pneumonia lesions. Coloured bars represent medians and black bars represent interquartile ranges.

Figure 5A). There were no significant differences in mass of pneumonia lesions between male patients (median values, 49,576; IQR, 6,679-145,001) and female patients (median values, 51,826; IQR, 11,392-124,774) $(\mathrm{P}=0.679$, Figure 5B).

\section{Discussion}

In this study, we systematically described dynamic longitudinal changes of COVID-19 pneumonia by quantitatively calculating mass of pulmonary abnormalities in 581 patients with 1,309 chest CT examinations. To our knowledge, our study is the first study in which mass was used as an approach to measure changes of pulmonary involvement in patients with COVID-19. Our findings demonstrated that pulmonary abnormalities increased quickly after the onset of initial symptoms, and peaked on week 2. In more detail, peak of pulmonary involvement occurred on day 10 after the onset of initial symptoms, and then gradually declined. Furthermore, pulmonary involvement of older patients ( $>45$ years) peaked later than that of younger patients ( $\leq 45$ years) (day $11 v s$. day 8 ). And pulmonary involvement in older patients ( $>45$ years) was significantly severer than that of younger patients ( $\leq 45$ years). In addition, there were no significant differences in the temporal changes of pulmonary involvement between male and female patients.

Several studies reported temporal changes of CT findings in COVID-19 pneumonia by subjectively observing imaging findings in CT images. A previous study described the characteristic CT findings of COVID-19 in five temporal stages, including ultra-early stage, early stage, rapid progression stage, consolidation stage, and dissipation stage (3). In the ultra-early stage (asymptomatic, 1-2 weeks after exposure), CT findings may include single or scattered focal GGO and patchy consolidation (3). In the early stage (early symptomatic presentation), CT findings may include single or scattered patchy or agglomerated GGO. In the rapid progression stage (days 3-7 after symptoms onset), CT may show a fused and large-scale light consolidation because of the cell-rich exudation in the alveolar cavity (3). In the consolidation stage (2nd week of symptoms onset), CT may show multiple patchy consolidations due to the fibrous exudation in the alveolar cavity. During the dissipation stage (2-3 weeks after symptoms onset), CT may show dispersed patchy consolidation or strip-like opacity. In addition, Pan et al. (2) investigated the temporal course of CT changes in 21 COVID-19 patients. During early stage, most patients showed more GGO and a less involved lobes relative to the later follow-up examinations. Nevertheless, increased crazy-paving pattern, increased involved lobes number, and appearance of consolidations were found in most patients over time (2). Further, their findings showed that lung abnormalities were greatest severity approximately on day 10 after the onset of initial symptoms. After day 14, improvement in imaging signs such as reduced involved lobes number and dissipation of crazy-paving pattern and consolidations occurred in $3 / 4$ patients (2). In another study, Wang et al. (6) investigated the temporal changes of CT findings in 90 COVID-19 patients. They found that the extent of pulmonary involvement peaked during days 6-11 after the onset of initial symptoms (6). However, all the above studies about the temporal changes of COVID-19 pneumonia were investigated just by subjectively observing imaging findings rather than quantitatively computing pulmonary involvement on CT images. Consistent with these reports $(2,6)$, our multi-center, large sample study 
also showed pulmonary involvement peaked on day 10 after the onset of initial symptoms. To our knowledge, our study is the first study in which mass was used as an approach to measuring change of pulmonary involvement using AI-based quantitative CT measurements in patients with COVID-19 pneumonia. Our study suggests that AI-based quantitative mass evaluation for COVID-19 pneumonia hold great potential for monitoring the disease progression.

Song et al. (18) reported the imaging findings in 51 patients with COVID-19 pneumonia. They found that GGO was present in $77 \%$ and consolidations were reported in $23 \%$ of younger patients. However, GGO in $55 \%$ and consolidations in $45 \%$ of older patients were reported. The older patients had more consolidation lesions than younger patients $(\mathrm{P}<0.001)(18)$. Additionally, uncharacteristic imaging signs were also present more commonly in older patients with COVID-19 pneumonia (3). In this study, we further found mass of pulmonary involvement of older patients was severer than that of younger patients. One possible reason for the more exudation in older patients is that the elderly have higher levels of angiotensin converting enzyme 2 in their lung alveoli (19), which is regarded as the receptor for SARS-Cov-2. Further, we found pulmonary involvement of older patients peaked later than that of younger patients. Recently, Zheng et al. (20) evaluated the correlation between age and duration of virus. They found that virus duration was longer in older patients than younger patients (20), which is partly due to immunosenescence (21). Considering sex-based differences in susceptibility to SARS$\mathrm{CoV}$ infection due to the differences in hormone levels (22), in this study, we further investigated the differences in temporal changes of pulmonary involvement between male and female patients. However, there were no significant differences in both the peak time and mass of pulmonary involvement between male and female.

This study has a number of strengths, including the multi-center, the large sample size, and the AI-based quantitative evaluation. However, several limitations in our study should be noted, such as the retrospective nature of our study and lack of a severe COVID-19 comparison subgroup. Subgroup comparison analysis would contribute to a more comprehensive understanding of the disease progression. In the future, prospective subgroup cohort analysis of mild and severe patients should be performed.

In conclusion, pulmonary abnormalities peaked on day 10 after the onset of initial symptoms in patients with COVID-19 pneumonia. Further, pulmonary involvement of older patients was severer and peaked later (day 11 $v s$. day 8) than that of younger patients. However, there were no significant differences in the temporal changes of pulmonary involvement between male and female patients. To our knowledge, this study is the first study in which mass was used as an approach to measure dynamic changes of COVID-19 pneumonia using AI-based quantitative CT measurements. These findings suggest that AI-based quantitative mass evaluation of COVID-19 pneumonia hold great potential for monitoring the disease progression.

\section{Acknowledgments}

Funding: This research was supported by Zhejiang University special scientific research fund for COVID-19 prevention and control (No. 2020XGZX036).

\section{Footnote}

Reporting Checklist: The authors have completed the STROBE reporting checklist. Available at http://dx.doi. org/10.21037/atm-20-4004

Data Sharing Statement: Available at http://dx.doi. org/10.21037/atm-20-4004

Conflicts of Interest: All authors have completed the ICMJE uniform disclosure form (available at http://dx.doi. org/10.21037/atm-20-4004). The authors have no conflicts of interest to declare.

Ethical Statement: The authors are accountable for all aspects of the work in ensuring that questions related to the accuracy or integrity of any part of the work are appropriately investigated and resolved. The study was conducted in accordance with the Declaration of Helsinki (as revised in 2013). This retrospective multi-center study was approved by the Institutional Review Board of each participating hospital. The written informed consent was waived.

Open Access Statement: This is an Open Access article distributed in accordance with the Creative Commons Attribution-NonCommercial-NoDerivs 4.0 International License (CC BY-NC-ND 4.0), which permits the noncommercial replication and distribution of the article with the strict proviso that no changes or edits are made and the original work is properly cited (including links to both the formal publication through the relevant DOI and the license). 
See: https://creativecommons.org/licenses/by-nc-nd/4.0/.

\section{References}

1. Huang C, Wang Y, Li X, et al. Clinical features of patients infected with 2019 novel coronavirus in Wuhan, China. Lancet 2020;395:497-506.

2. Pan F, Ye T, Sun P, et al. Time Course of Lung Changes at Chest CT During Recovery From Coronavirus Disease 2019 (COVID-19). Radiology 2020;295:715-21.

3. Jin $\mathrm{YH}$, Cai L, Cheng ZS, et al. A rapid advice guideline for the diagnosis and treatment of 2019 novel coronavirus (2019-nCoV) infected pneumonia (standard version). Mil Med Res 2020;7:4.

4. Shi H, Han X, Jiang N, et al. Radiological findings from 81 patients with COVID-19 pneumonia in Wuhan, China: a descriptive study. Lancet Infect Dis 2020;20:425-34.

5. Pan Y, Guan H, Zhou S, et al. Initial CT findings and temporal changes in patients with the novel coronavirus pneumonia (2019-nCoV): a study of 63 patients in Wuhan, China. Eur Radiol 2020;30:3306-9.

6. Wang Y, Dong C, Hu Y, et al. Temporal Changes of CT Findings in 90 Patients with COVID-19 Pneumonia: A Longitudinal Study. Radiology 2020:200843.

7. Fang Y, Zhang H, Xu Y, et al. CT Manifestations of Two Cases of 2019 Novel Coronavirus (2019-nCoV) Pneumonia. Radiology 2020;295:208-9.

8. Lei J, Li J, Li X, et al. CT Imaging of the 2019 Novel Coronavirus (2019-nCoV) Pneumonia. Radiology 2020;295:18.

9. Shi H, Han X, Zheng C. Evolution of CT Manifestations in a Patient Recovered from 2019 Novel Coronavirus (2019-nCoV) Pneumonia in Wuhan, China. Radiology 2020;295:20.

10. Lee HJ, Goo JM, Lee CH, et al. Nodular groundglass opacities on thin-section CT: size change during follow-up and pathological results. Korean J Radiol 2007;8:22-31.

11. Marten K, Auer F, Schmidt S, et al. Inadequacy of manual measurements compared to automated CT volumetry in assessment of treatment response of pulmonary metastases using RECIST criteria. Eur Radiol 2006;16:781-90.

12. Mull RT. Mass estimates by computed tomography: physical density from CT numbers. AJR Am J Roentgenol 1984;143:1101-4.
13. de Hoop B, Gietema H, van de Vorst S, et al. Pulmonary ground-glass nodules: increase in mass as an early indicator of growth. Radiology 2010;255:199-206.

14. Ardila D, Kiraly AP, Bharadwaj S, et al. End-to-end lung cancer screening with three-dimensional deep learning on low-dose chest computed tomography. Nat Med 2019;25:954-61.

15. Li L, Qin L, Xu Z, et al. Artificial Intelligence Distinguishes COVID-19 from Community Acquired Pneumonia on Chest CT. Radiology 2020:200905.

16. Liu F, Zhang Q, Huang C, et al. CT quantification of pneumonia lesions in early days predicts progression to severe illness in a cohort of COVID-19 patients. Theranostics 2020;10:5613-22.

17. Wang S, Zhou M, Liu Z, et al. Central focused convolutional neural networks: Developing a data-driven model for lung nodule segmentation. Med Image Anal 2017;40:172-83.

18. Song F, Shi N, Shan F, et al. Emerging 2019 Novel Coronavirus (2019-nCoV) Pneumonia. Radiology 2020;295:210-7.

19. Chen Y, Shan K, Qian W. Asians Do Not Exhibit Elevated Expression or Unique Genetic Polymorphisms for ACE2, the Cell-Entry Receptor of SARS-CoV-2. Preprints 2020:2020020258.

20. Zheng S, Fan J, Yu F, et al. Viral load dynamics and disease severity in patients infected with SARS-CoV-2 in Zhejiang province, China, January-March 2020: retrospective cohort study. BMJ 2020;369:m1443.

21. Pera A, Campos C, Lopez N, et al. Immunosenescence: Implications for response to infection and vaccination in older people. Maturitas 2015;82:50-5.

22. Channappanavar R, Fett C, Mack M, et al. SexBased Differences in Susceptibility to Severe Acute Respiratory Syndrome Coronavirus Infection. J Immunol 2017;198:4046-53.

Cite this article as: Wang C, Huang P, Wang L, Shen $Z$, Lin B, Wang Q, Zhao T, Zheng H, Ji W, Gao Y, Xia J, Cheng J, Ma J, Liu J, Liu Y, Su M, Ruan G, Shu J, Ren D, Zhao Z, Yao W, Yang Y, Liu B, Zhang M. Temporal changes of COVID-19 pneumonia by mass evaluation using CT: a retrospective multi-center study. Ann Transl Med 2020;8(15):935. doi: 10.21037/atm-20-4004 


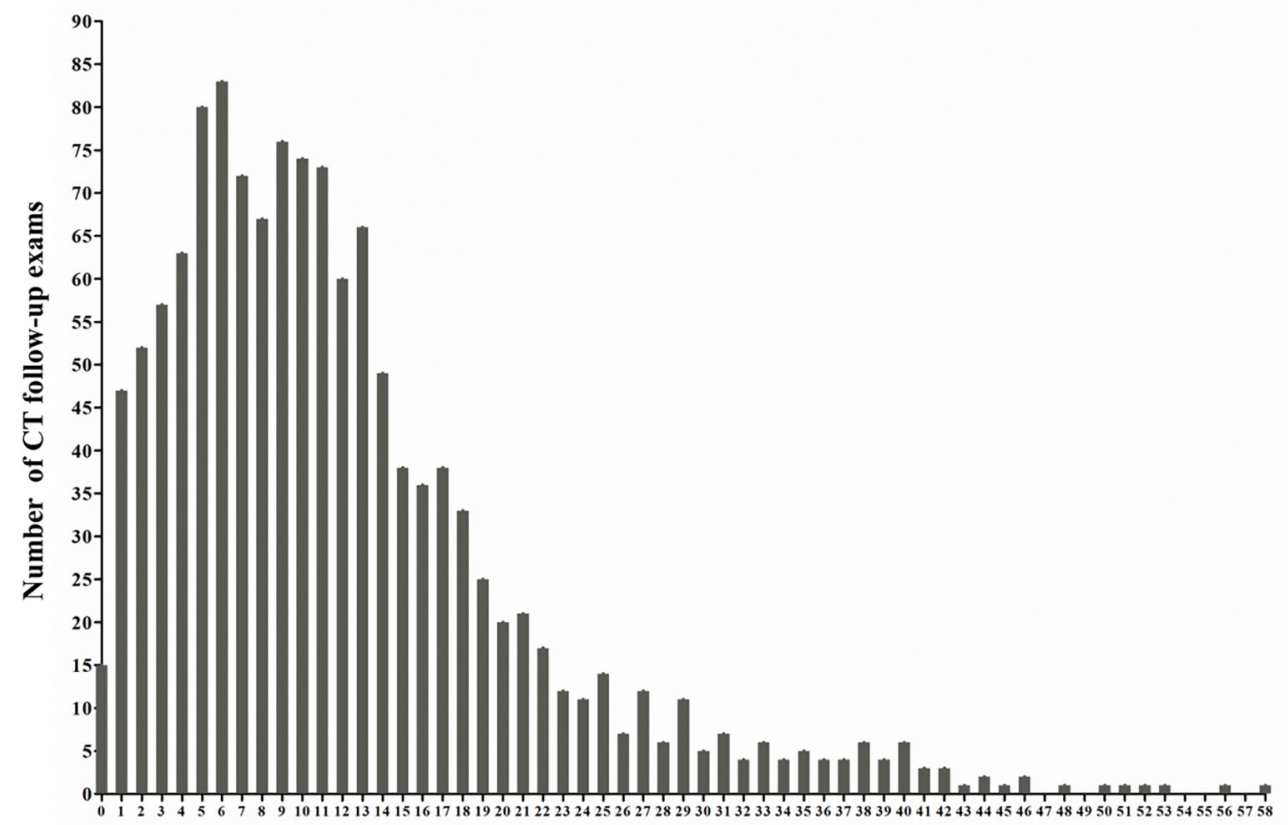

Time from the onset of initial symptoms (days)

Figure S1 The number distribution of CT follow-up exams.

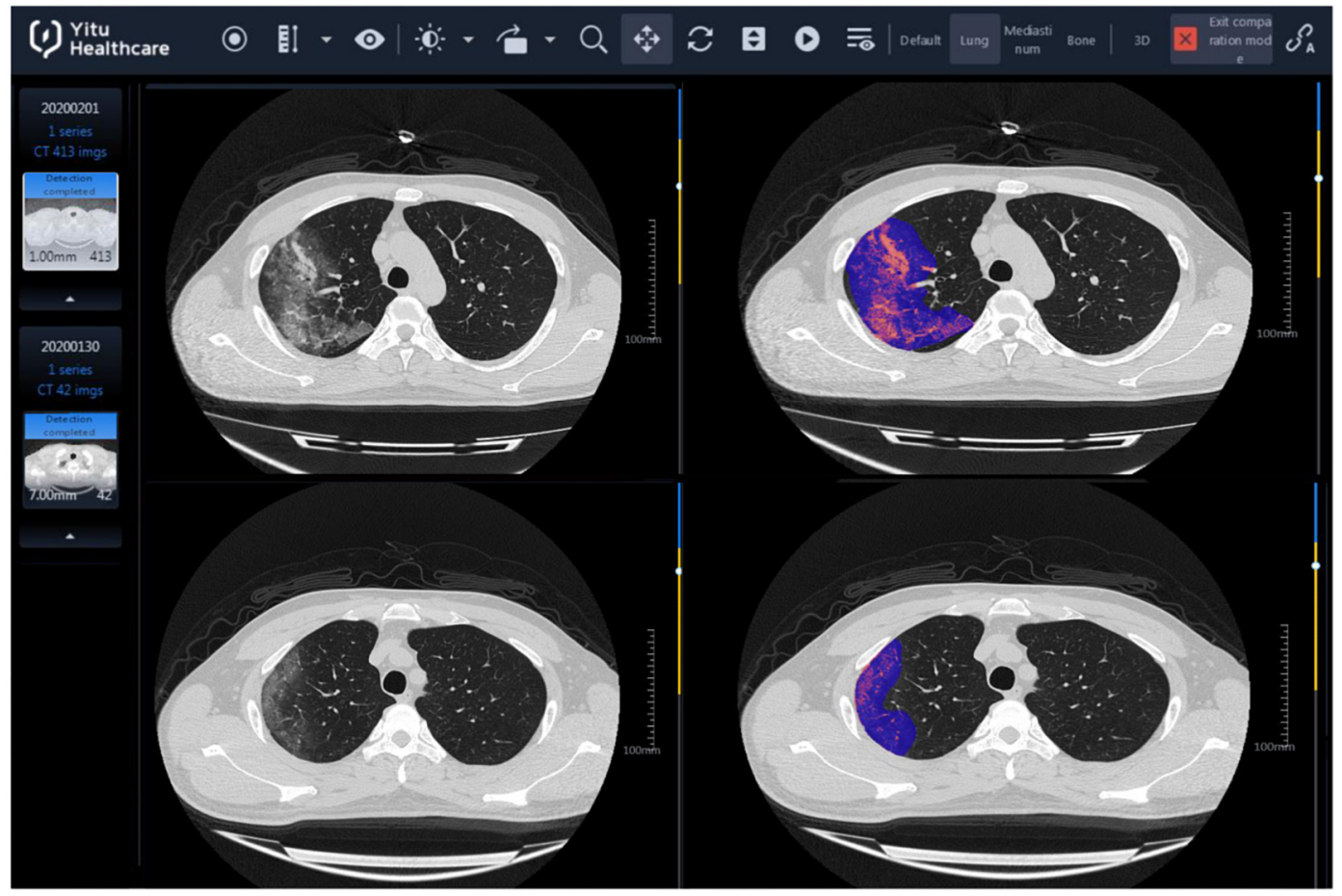

Figure S2 The quantitative evaluation system of CT for COVID-19 with artificial intelligence (AI). COVID-19, coronavirus disease 2019. 